\title{
Educação médica: linha do tempo e panorama da pós-graduação stricto sensu no Brasil
}

Medical education: timeline and panorama of stricto sensu graduate studies in Brazil

\author{
Hayslla Boaventura Piotto1,2 (D) haysllabp@gmail.com \\ Luciana Calabró ${ }^{1}$ (1) $\mid$ luciana.calabro@ufrgs.br
}

\section{RESUMO}

Introdução: O presente trabalho apresenta uma sucinta retrospectiva da pós-graduação stricto sensu brasileira e elucida o atual panorama quantitativo das subáreas avaliativas da área de medicina.

Objetivo: Este estudo teve como objetivo esboçar um panorama crítico da pós-graduação médica.

Método: O método, aqui utilizado, contempla uma revisão das normativas que conduziram a formação da pós-graduação stricto sensu brasileira e uma categorização e análise estatística de dados fornecidos pela Coordenação de Aperfeiçoamento de Pessoal de Nível Superior, complementados por informações da Plataforma Sucupira e do último censo do Instituto Brasileiro de Geografia e Estatística, com recorte das áreas avaliativas da medicina e atenção a elas.

Resultado: Como resultado, observou-se que, em junho de 2019, o Brasil atingiu 4.590 programas de pós-graduação, sendo 262 distribuídos nas áreas avaliativas da medicina. A partir desse quantitativo, apresentam-se uma tabela, dois quadros, uma figura e sete gráficos que demostram diferentes aspectos do Sistema Nacional de Pós-Graduação.

Conclusão: Em conclusão, no que se refere ao Sistema Nacional de Pós-Graduação, descrevem-se, como ponto forte, a expansão e o crescimento da pós-graduação, e indicam-se, como ponto frágil, os traços de assimetrias regionais. Em face das subáreas da medicina, são evidenciadas as características de cada área avaliativa, incluindo relações entre modalidades, níveis e conceitos dos programas de pós-graduação. Por fim, fica a perspectiva de que, além do número de programas de pós-graduação e de seus respectivos conceitos, um panorama qualitativo deve considerar indicadores como produção científica e impacto social, temas a serem abordados em estudos futuros, utilizando-se dados e as ferramentas metodológicas específicas.

Palavras-chave: Educação Médica; Educação de Pós-Graduação em Medicina; Avaliação Educacional.

\begin{abstract}
Introduction: This work offers a succinct retrospective of the Brazilian stricto sensu graduate program and elucidates the current quantitative panorama of the evaluative sub-areas of Medicine.

Objective: This study aimed to outline a critical panorama of medical Graduate Program.

Method: The method addressed here includes a review of the regulations that led to the formation of the Brazilian stricto sensu graduate programs and a categorization and statistical analysis of data provided by CAPES (Coordination for the Improvement of Higher Education Personnel), complemented by information from the Sucupira Platform and the latest census published by the Brazilian Institute of Geography and Statistics, focusing on the evaluative areas of Medicine.

Result: It was observed that in June 2019, Brazil reached 4,590 Graduate Programs, of which 262 programs were distributed in the evaluative areas of Medicine. Based on this quantity, tables, images and graphs are presented that show different aspects of the National Graduate System.

Conclusion: With regard to the National Graduate System, the expansion and growth of graduate studies are described as a strong point, whereas the characteristics of regional asymmetries are indicated as a shortcoming. In view of the sub-areas of medicine, the characteristics of each evaluation area are highlighted, including relationships between modalities, levels and Graduate Program, and scores. Finally, the view persists that, in addition to the number of Graduate Program, and their respective scores, a qualitative panorama should consider indicators such as scientific production and social impact, themes to be addressed in future studies, using specific methodological data and tools.
\end{abstract}

Keywords: Education Medical; Graduate Education in Medicine; Educational Measurement.

${ }^{1}$ Universidade Federal do Rio Grande do Sul, Porto Alegre, Rio Grande do Sul, Brasil.

${ }^{2}$ Coordenação de Aperfeiçoamento de Pessoal de Nível Superior, Brasília, Distrito Federal, Brasil.

Editora-chefe: Daniela Chiesa

Editora associada: Daniela Chiesa

Recebido em 28/02/20; Aceito em 24/12/20.

Avaliado pelo processo de double blind review. 


\section{INTRODUÇÃO: UM BREVE HITÓRICO}

O início da educação médica brasileira data de 1808, quando Dom João VI instaura em Salvador e no Rio de Janeiro as primeiras Faculdades de Medicina do Brasil. Até então, a formação oficial de médicos ocorria fora do país, especialmente em Lisboa.

Aberta em 18 de fevereiro de 1808, via carta régia, a Escola de Medicina e Cirurgia no Hospital Militar da Bahia configurou-se como a primeira escola de ensino superior no Brasil, e pouco depois, em 2 de abril do mesmo ano, era inaugurada a Escola Cirúrgica do Rio de Janeiro. Começava, assim, a educação superior nacional (p. 67)'.

Considerando as dificuldades da formação médica em outro continente e o pequeno universo de profissionais diplomados atuantes em território brasileiro, o atendimento "médico" massivo da população brasileira à época ocorria, em via de regra, por profissionais sem a devida qualificação/ habilitação, os conhecidos curandeiros e cirurgiões barbeiros. Em 1828, foi determinado que apenas "médicos diplomados podiam clinicar" (p. 162)2, e a partir de então a educação superior do Brasil sofreu grandes modificações e significativa expansão. Especificamente, a educação médica passou por várias reformulações curriculares, porém, até meados do século $X X$, esse ensino, mesmo com todas as suas modificações/ evoluções, seguiu dissociado da pesquisa. Nesse sentido, de acordo com o Plano Nacional de Pós-Graduação (PNPG), período 2011-2020, "o surgimento das Faculdades de Medicina teve grande influência na área de formação de recursos humanos e no desenvolvimento das diversas especializações"(214) 3 .

Deixando de lado a graduação e com direcionamento para a pós-graduação stricto sensu, que é o foco desta pesquisa, quando se percorre o marco legal da história da educação no Brasil, observa-se que a primeira referência normativa brasileira relacionada ao curso de doutorado ocorreu em 1931, com os decretos nos $19.851^{4}$ e $19.852^{5}$.

[...] a ideia de cursos de doutorado surge, pela primeira vez, com a Reforma Francisco Campos, em 1931. O Estatuto das Universidades Brasileiras (Decreto $n^{\circ}$ 19.851, de 11 de abril de 1931) previa o doutoramento de tipo europeu. [...] Por sua vez, o Decreto no 19.852, também de 11 de abril de 1931, que dispunha sobre a organização da Universidade do Rio de Janeiro, criava cursos regulares de doutorado no campo do direito e das ciências exatas e naturais. Tais cursos constituíam, de fato, uma pós-graduação, que hoje denominamos stricto sensu (p. 3) 6 .

Apesar da "legalização" do curso de doutorado e da observação de Newton Sucupira sobre a pós-graduação stricto sensu, o Decreto no 19.852 trouxe a formação médica com características de pós-graduação lato sensu, o que hoje seria equivalente à residência médica, pois, quando se conferem os artigos específicos dele, é possível observar que atividades de investigação e pesquisa, que são essenciais na pós-graduação stricto sensu, não foram descritas na normativa.

Art. 57. O ensino médico será ministrado nos cursos abaixo definidos:

a) cursos normais, seriados, que se destinam ao ensino das disciplinas essenciais ao exercício da medicina prática, nos seus diversos rumos, e serão regidos pelos professores das respectivas cadeiras;

b) cursos equiparados, que serão realizados pelos docentes livres de acordo com programas nos moldes dos cursos normais, e com os efeitos legais dos mesmos;

c) cursos livres, nos quais serão ministrados ensinamentos sobre as disciplinas do curso médico ou sobre assuntos científicos correlatos, mas que não têm os efeitos legais dos cursos anteriores;

d) cursos de aperfeiçoamento, destinados a ampliar conhecimentos em qualquer das disciplinas do curso médico ou em assuntos parciais das mesmas;

e) cursos de especialização, que se destinam a formar especialistas nos diversos ramos da medicina aplicada (grifo nosso) $^{5}$.

Mesmo com essa previsão legal, a expansão da educação superior no que tange à pós-graduação ocorreu no Brasil somente com o fim da era Vargas. A nomenclatura "pósgraduação" e a ideia de "ensino e pesquisa" foram normatizadas pela primeira vez em 1946 a partir do Decreto $n^{\circ} 21.321^{7}$ Estatuto da Universidade do Brasil:

Art. $2^{\circ}$ Os objetivos da Universidade do Brasil abrangem a educação, o ensino e a pesquisa.

[...]

Art. 71. Os cursos universitários serão os seguintes:

a) cursos de formação;

b) cursos de aperfeiçoamento;

c) cursos de especialização;

d) cursos de extensão;

e) cursos de pós-graduação;

f) cursos de doutorado.

Na mesma época, o fim da Segunda Guerra Mundial mostrou ao mundo o poder da pesquisa, da ciência e da tecnologia, e, no Brasil, Anísio Teixeira já galgava os pilares para a criação da Coordenação de Aperfeiçoamento de Pessoal de 
Nível Superior (Capes) e para a consolidação da pós-graduação stricto sensu.

A CAPES foi criada pelo Decreto 29.741, de 11 de julho de 1951, que Institui[u] uma Comissão para promover a Campanha Nacional de Aperfeiçoamento de Pessoal de Nível Superior. Anísio Teixeira foi então convidado pelo Ministro da Educação e Saúde, à época, Ernesto Simões Filho, e assumiu o cargo de Secretário Geral da Comissão que tinha seis meses como prazo para a instalação da Campanha - prazo, posteriormente, dilatado para um ano pelo Decreto $30.286\left(\right.$ p. 115) ${ }^{8}$.

Desde então, as universidades começaram a ofertar cursos de mestrado e doutorado, e o primeiro Programa de Pós-Graduação (PPG) na área da medicina, registrado no Sistema Nacional de Pós-Graduação (SNPG), data de 1959, na Universidade Federal de Minas Gerais: o curso de pósgraduação em Oftalmologia da Faculdade de Medicina.

Na década de 1960, houve um forte movimento de expansão das instituições de ensino superior (IES) no Brasil, o que veio a coincidir com a publicação da Lei de Diretrizes e Bases da Educação Nacional (LDB), Lei no 4.024', de 20 de dezembro de 1961, normativa precursora dos segmentos da educação superior:

Art. 69. Nos estabelecimentos de ensino superior podem ser ministrados os seguintes cursos:

a) de graduação, abertos à matrícula de candidatos que hajam concluído o ciclo colegial ou equivalente, $e$ obtido classificação em concurso de habilitação;

b) de pós-graduação, abertos a matrícula de candidatos que hajam concluído o curso de graduação e obtido o respectivo diploma;

c) de especialização, aperfeiçoamento e extensão, ou quaisquer outros, a juízo do respectivo instituto de ensino abertos a candidatos com o preparo e os requisitos que vierem a ser exigidos (grifo nosso).

Apesar de um histórico de mais de 200 anos de educação superior, o primeiro documento para a determinação dos parâmetros da pós-graduação brasileira foi publicado em dezembro de 1965, o Parecer no $977 / 65^{10}$ do Conselho Federal de Educação (CFE), conhecido como Parecer Newton Sucupira. Além da inspiração na pós-graduação norte-americana, esse documento apresentou a definição da natureza e dos objetivos dos cursos de pós-graduação, como uma espécie de regulamentação que à época também possibilitou "distinguir a pós-graduação sensu stricto e sensu lato".

Em resumo, a pós-graduação sensu stricto apresenta as seguintes características fundamentais: é de natureza acadêmica e de pesquisa e mesmo atuando em setores profissionais tem objetivo essencialmente científico, enquanto a especialização, via de regra, tem sentido eminentemente prático-profissional; confere grau acadêmico e a especialização concede certificado; finalmente a pós-graduação possui uma sistemática formando estrato essencial e superior na hierarquia dos cursos que constituem o complexo universitário. Isto nos permite apresentar o seguinte conceito de pós-graduação sensu stricto: o ciclo de cursos regulares em segmento à graduação, sistematicamente organizados, visando desenvolver e aprofundar a formação adquirida no âmbito da graduação e conduzindo à obtenção de grau acadêmico ${ }^{10}$.

Até hoje, o Parecer Sucupira é um documento referencial para tratar da pós-graduação brasileira, mas não suficiente, uma vez que ele apenas indica a necessidade das "normas fixando os critérios de aprovação dos cursos", função adotada pela Capes a partir da década de 1970, quando o professor doutor Darcy Closs

[...] trouxe para CAPES, como referência, sua experiência na Universidade Federal do Rio Grande Sul na década de 1960 e suas visitas técnicas aos sistemas de avaliação nos EUA e na Alemanha, [...] um processo embrionário de avaliação dos programas de pós-graduação foi implantado primeiro nos cursos novos. Por indicação do professor Darcy, antes do encaminhamento ao Conselho Federal de Educação, os projetos passavam pelo crivo de um Comitê de especialistas composto por docentes da comunidade acadêmica, formando, assim, uma teia de relações. Como funciona até hoje, o Conselho passou, na época, uma resolução: "a partir de hoje a CAPES primeiro examina e nós só vamos chancelar aquilo que foi aprovado pelos Comitês Assessores da CAPES" (p. 299) ${ }^{11}$.

Surge assim o processo de avaliação e acompanhamento da pós-graduação, delegado à Capes pela Lei no $8.405^{12}$, de 9 de janeiro de 1992:

Art. $2^{\circ}$ A Fundação CAPESterácomofinalidadesubsidiar - Ministério da Educação na formulação de políticas para a área de pós-graduação, coordenar e avaliar os cursos desse nível no País e estimular, mediante bolsas de estudo, auxílios e outros mecanismos, a formação de recursos humanos altamente qualificados para a docência de grau superior, a pesquisa e o atendimento da demanda dos setores públicos e privado.

Com o objetivo de esboçar um panorama crítico da pósgraduação médica, o presente trabalho é composto de uma revisão das normativas que conduziram a formação da pósgraduação brasileira e uma categorização e análise estatística de dados do SNPG, com recorte das áreas avaliativas da medicina e atenção a elas. 


\section{MÉTODOS}

Para apresentar um panorama da pós-graduação médica no Brasil, além do arranjo de artigos científicos e normativas utilizados para traçar a linha do tempo exposta na seção anterior, o presente artigo tem como método uma análise quantitativa de diferentes características dos PPGs de Medicina, associadas a aspectos do SNPG e a notas do sistema avaliativo até então praticado pela Capes.

Foi utilizado um relatório analítico sobre o funcionamento dos PPGs brasileiros, em formato .xlsx, fornecido pela Divisão de Supervisão de Resultados (DSR) da Diretoria de Programas e Bolsas (DPB) - Capes. O relatório foi disponibilizado em junho de 2019 após solicitação formal via ofício e é composto de informações relativas aos PPGs já registrados na Capes desde 1931.

Por se tratar de uma planilha muito extensa, com 5.285 códigos de PPGs distintos e mais de 50 colunas informativas, realizou-se uma filtragem utilizando os seguintes parâmetros: código do PPG, grande área, colégio, área de avaliação, área do conhecimento, nome do programa, modalidade (acadêmico ou profissional), situação (em projeto, em funcionamento, em desativação e desativado), nível (mestrado ou doutorado) e conceito do PPG (1 a 7 ou A). Em seguida, houve uma consulta à Plataforma Sucupira a fim de coletar dados como nome e sigla das IES, natureza jurídica delas (particular, pública federal, pública estadual ou pública municipal) e distribuição geográficas das instituições (unidade federativa). A partir desses parâmetros, foram feitas categorizações que permitiram a demonstração de estruturas, quantidades, evolução, distribuição e fração dos dados por meio de uma tabela, dois quadros, uma figura e sete gráficos, e também se apresenta uma breve análise que leva em conta a distribuição dos PPGs por unidade federativa (UF) e o Índice de Desenvolvimento Humano (IDH) de cada região, embasados no panorama do último censo do Instituto Brasileiro de Geografia e Estatística $(\mathrm{IBGE})^{13}$. Aqui, foi tomado como base o estudo realizado por João Evangelista Steiner, "Qualidade e diversidade institucional na pós-graduação brasileira”, publicado em $2005^{14}$.

\section{RESULTADOS E DISCUSSÃO}

\section{A Capes e a pós-graduação médica}

Entre seus pontos de atuação, a Capes tem ênfase no cenário nacional com avaliação e acompanhamento da pós-graduação stricto sensu, mas também atua na formação de recursos humanos de alto nível no país e no exterior, no acesso, na divulgação e na cooperação científica nacional e internacional, e na indução e no fomento da formação de professores para a educação básica. Tais atribuições foram estabelecidas pela Lei no $11.502^{15}$, de 11 de julho de 2007.

Quanto à avaliação da pós-graduação, o PNPG 2011-
$2020^{3}$ estabelece o seguinte:

Os índices propostos até então davam ênfase à produtividade dos orientadores e à participação do aluno formado na produção científica e tecnológica dos laboratórios ou grupos de pesquisa que compõem a pós-graduação. Os índices deveriam refletir a relevância do conhecimento novo, sua importância no contexto social e o impacto da inovação tecnológica no mundo globalizado e competitivo ( $p$. 36).

No cumprimento dessa missão, a Capes vem realizando e aprimorando o exercício avaliativo desde 1976. As primeiras avaliações foram realizadas por meio de consultores externos com emissão de conceitos:

[...] aconteceu a primeira avaliação do Sistema Nacional de Pós-Graduação (SNPG). Foi adotada uma escala de cinco conceitos ( $A$, avaliação mais alta, $B$, $C, D, E)$, e os cursos de mestrado e doutorado foram conceituados separadamente. Ficou instituída uma avaliação anual (p. 299)"1.

No período de 1976-1997, vigorou na classificação a escala conceitual alfabética de $A$ a $E$, sendo considerados cursos de padrão internacional aqueles contemplados com o conceito A. A partir de 1997, passou a vigorar a escala numérica de 1 a 7 ; sendo considerados cursos de padrão internacional aqueles classificados com os conceitos 6 e7, os cursos 7 ocupando o topo do sistema (p.125) ${ }^{3}$.

Depois disso, vieram a Resolução do Conselho Nacional de Educação e da Câmara de Educação Superior (CNE/CES) $n^{\circ} 1 / 2001^{16}$ e a Portaria Capes no $13 / 2002^{17}$. A partir daí, os cursos de mestrado e doutorado deixaram de ser considerados separadamente, as notas passaram a ser numéricas, numa escala de 1 a 7, e o resultado da avaliação passou a ser homologado por um conselho. Em 2005, na Portaria Capes no 99/2005 ${ }^{18}$, foi instituída a coleta de dados da pós-graduação para elaboração de indicadores e estatísticas. A finalidade desses indicadores é determinar a nota de cada curso e, por conseguinte, a aprovação de cursos novos e os critérios para continuidade dos cursos já em andamento. Atualmente, a avaliação ocorre em períodos quadrienais - Portaria Capes n 59/2017 ${ }^{19}$.

Para organizar essa avaliação, as áreas do saber foram estruturadas em divisões e subdivisões com critérios afins e com protocolos avaliativos, em que são estruturados cinco quesitos básicos: 1. proposta do programa, 2. corpo docente, 3 . corpo discente, teses e dissertações, 4. produção intelectual e 5. inserção social. $O$ diferencial avaliativo ocorre entre as áreas, com a determinação de pesos/porcentagens distintos para cada quesito. $\mathrm{O}$ Quadro 1 demonstra a divisão das áreas do conhecimento em três níveis: colégios, grandes áreas e áreas de avaliação. 
Quadro 1. Listas das 49 áreas de avaliação organizadas por colegiados e grandes áreas.

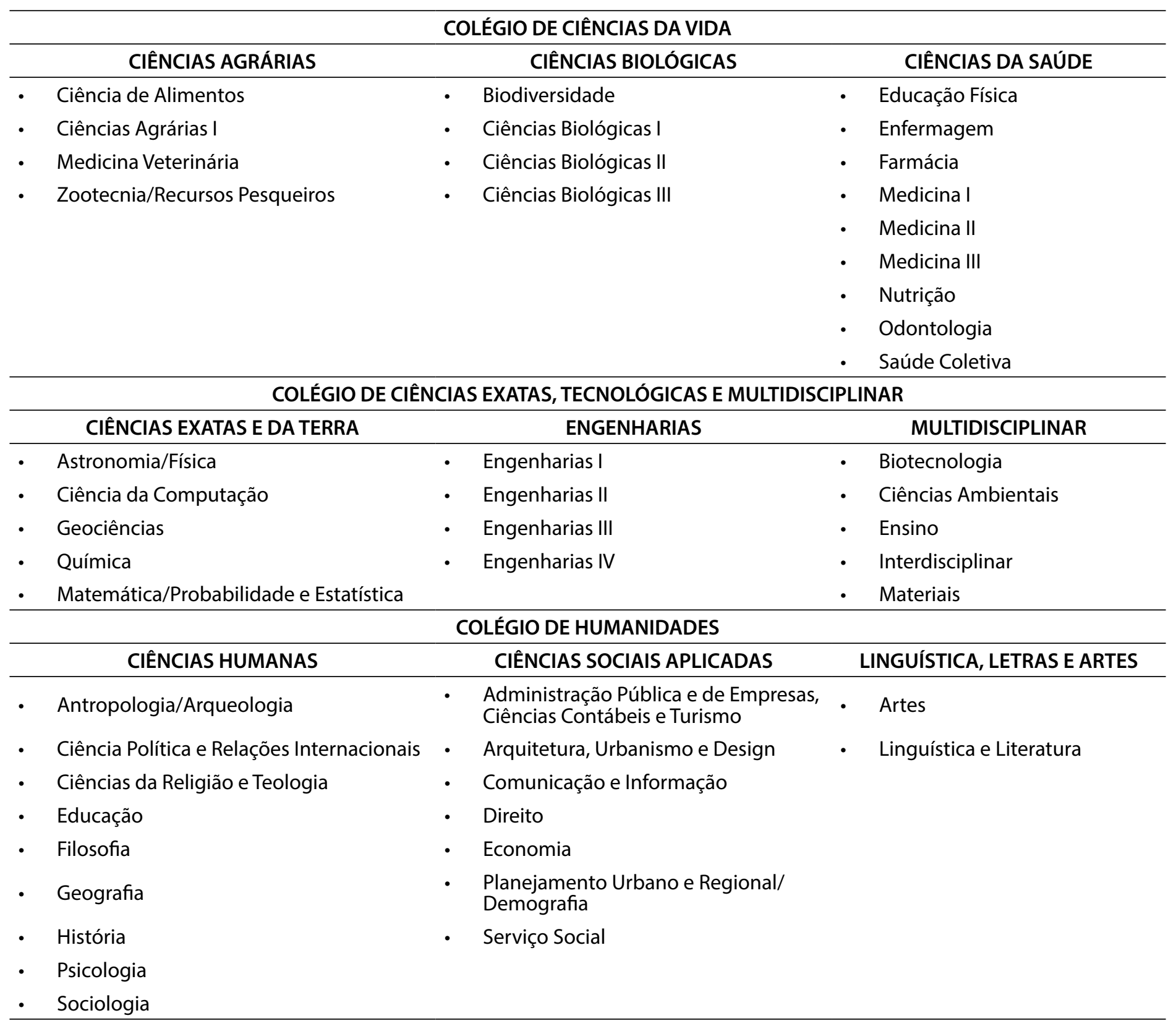

Fonte: Adaptado pelas autoras com base no site da Capes $^{20}$.

Cada uma das 49 áreas de avaliação possui um coordenador que conduz os trâmites avaliativos e também possui documentos que descrevem as especificidades de cada área na avaliação:

\section{Neles estão descritos o estado atual, as características e as perspectivas, assim como os quesitos considerados prioritários na avaliação dos programas de pós- graduação pertencentes a cada uma das 49 áreas de avaliação. \\ Em conjunto com as Fichas de Avaliação e os Relatórios de Avaliação, os Documentos de Área constituem o trinômio que expressa os processos e os resultados da Avaliação Quadrienal20.}

A formação médica stricto sensu no Brasil está estruturada da seguinte maneira: colégio: Ciências da Vida; grande área: Ciências da Saúde; e, por fim, áreas avaliativas: Medicina I, Medicina II e Medicina III (Quadro 2). Como a medicina é um grande campo, com várias ramificações, ela está dividida em três áreas de avaliação fazendo um agrupamento de especialidades.

\section{Um panorama da pós-graduação médica}

Apresentadas as principais características estruturais da avaliação realizada pela Capes e das áreas avaliativas da medicina, faz-se significativo expor uma perspectiva mais técnica sobre a pós-graduação médica stricto sensu. Com base em dados extraídos da Plataforma Sucupira, associados a dados fornecidos pela DSR/DPB/Capes, foi possível ilustrar esse panorama. 
Quadro 2.Especialidades de cada área de avaliação da medicina.

\begin{tabular}{ll}
\hline SUBÁREA & \multicolumn{1}{c}{ DOMÍNIO DO CONHECIMENTO } \\
\hline & CANCEROLOGIA \\
& CARDIOLOGIA \\
& CLÍNICA MÉDICA \\
& DERMATOLOGIA \\
MEDICINA I & ENDOCRINOLOGIA \\
& GASTROENTEROLOGIA \\
& MEDICINA \\
& NEFROLOGIA \\
& PNEUMOLOGIA \\
& ALERGOLOGIA E IMUNOLOGIA CLÍNICA \\
& ANATOMIA PATOLÓGICA E PATOLOGIA \\
& CLÍNICA \\
& DOENÇAS INFECCIOSAS E PARASITÁRIAS \\
& HEMATOLOGIA \\
& NEUROLOGIA \\
& PEDIATRIA \\
& PSIQUIATRIA \\
& RADIOLOGIA MÉDICA \\
& REUMATOLOGIA \\
SAÚDE MATERNO-INFANTIL \\
ANEDICINASIOLOGIA \\
CIRURGIA \\
CIRURGIA EXPERIMENTAL \\
CIRURGIA OTORRINOLARINGOLOGIA \\
CIRURGIA PLÁSTICA E RESTAURADORA \\
CIRURGIA UROLÓGICA \\
GINECOLOGIA E OBSTETRÍCIA \\
OFTALMOLOGIA \\
ORTOPEDIA \\
\hline MEDICINA III
\end{tabular}

Fonte: Adaptado pelas autoras com base no site da Capes $^{20}$.

Em um cenário geral, em junho de 2019 o Brasil atingiu 4.590 programas de pós-graduação, o que demonstra um crescimento significativo com relação aos dados apresentados em 2010 pelo PNPG 2011-2020:

O panorama atual da pós-graduação brasileira congrega os seguintes dados: havia em 2009, 2.719 programas em atividade responsáveis por 4.101 cursos, sendo: 2.436 de mestrado (59,4\%); 1.422 de doutorado $(34,7 \%)$ e 243 de mestrado profissional $(5,9 \%)(p .45, \text { grifo nosso })^{3}$.

Geograficamente, a distribuição da pós-graduação brasileira por UF pode ser observada em um agrupamento de três distintos polos de concentração dos PPGs. A Figura 1 dispõe, em ordem crescente, a concentração de PPG por UF: o polo I, em tonalidade mais clara, com até 59 PPGs por estado; o polo II, em tonalidade média, é composto por estados que possuem entre 63 e 171 PPGs; e, por fim, em cinza-escuro, o polo III em que constam os estados com mais de 180 PPGs.

Outro arranjo relevante para esse panorama é a classificação dos PPGs por grande área. No Gráfico 1, podemos visualizar a distribuição desses 4.590 PPGs.

Figura 1. Distribuição dos PPGs por unidade federativa.

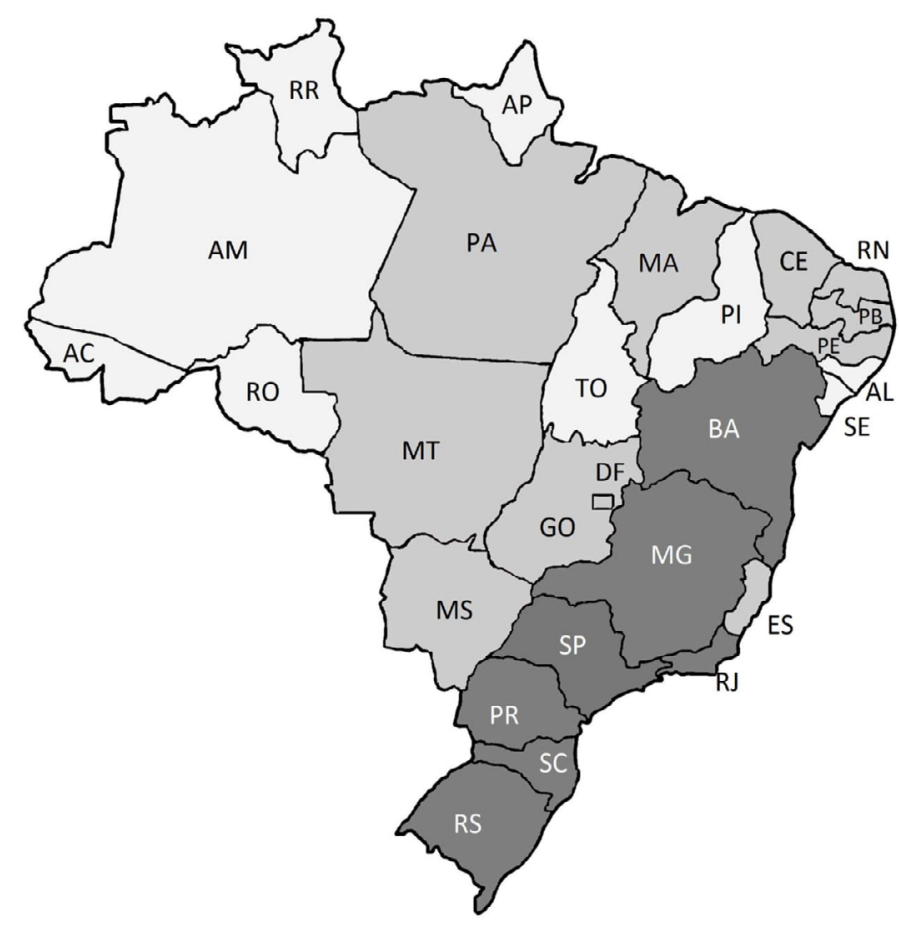

\begin{tabular}{|c|c|c|}
\hline UF & PPG & POLO \\
\hline Amapá & 10 & \multirow{9}{*}{ I } \\
\hline Roraima & 13 & \\
\hline Acre & 15 & \\
\hline Rondônia & 16 & \\
\hline Tocantins & 28 & \\
\hline Alagoas & 42 & \\
\hline Piauí & 46 & \\
\hline Sergipe & 56 & \\
\hline Amazonas & 59 & \\
\hline Maranhão & 63 & \multirow{11}{*}{ II } \\
\hline Mato Grosso & 65 & \\
\hline Espírito Santo & 75 & \\
\hline Mato Grosso do Sul & 79 & \\
\hline Rio Grande do Norte & 113 & \\
\hline Paraíba & 114 & \\
\hline Goiás & 117 & \\
\hline Distrito Federal & 125 & \\
\hline Pará & 132 & \\
\hline Ceará & 140 & \\
\hline Pernambuco & 171 & \\
\hline Santa Catarina & 183 & \multirow{7}{*}{ III } \\
\hline Bahia & 202 & \\
\hline Paraná & 369 & \\
\hline Rio Grande do Sul & 432 & \\
\hline Minas Gerais & 471 & \\
\hline Rio de Janeiro & 513 & \\
\hline São Paulo & 941 & \\
\hline
\end{tabular}


Gráfico 1.Distribuição dos PPGs por grande área.

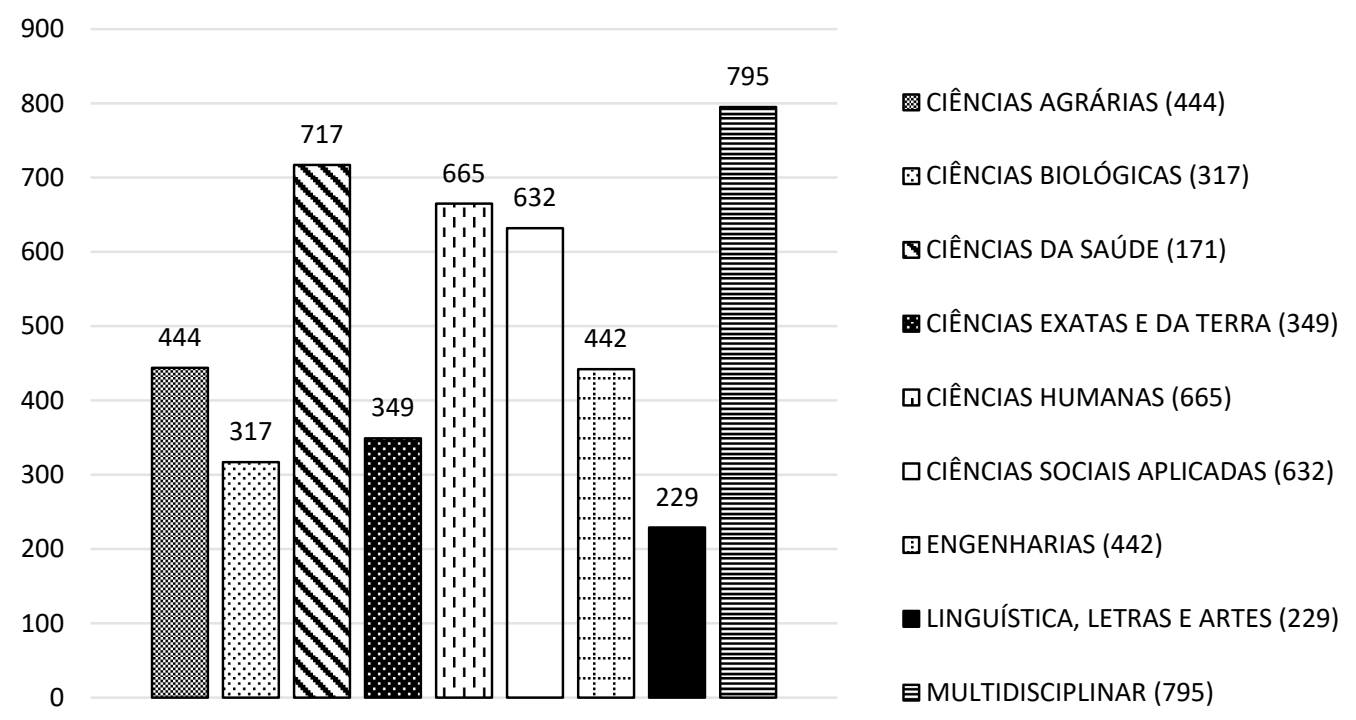

Gráfico 2.Distribuição dos PPGs da grande área Ciências da Saúde.

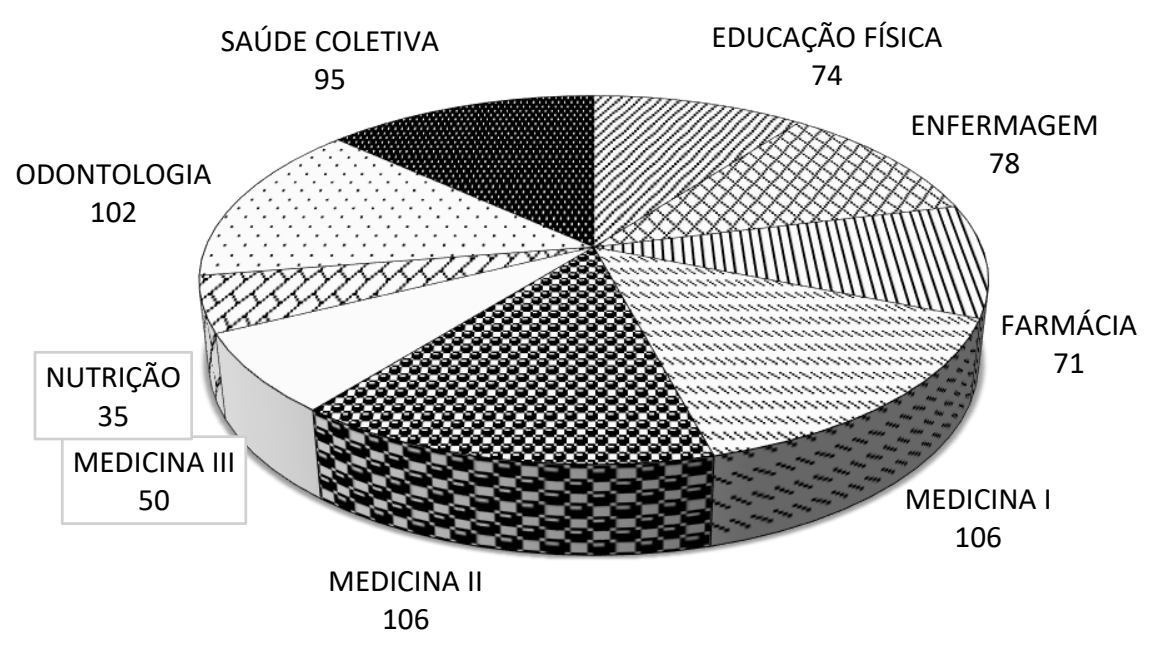

Notoriamente, as grandes áreas Multidisciplinar e Ciências da Saúde são as maiores do SNPG, com 17,5\% e $15,6 \%$, respectivamente, de representatividade no contexto nacional. Fazendo um recorte da grande área de interesse deste trabalho, a grande área das Ciências da Saúde possui 717 PPGs distribuídos em nove áreas de avaliação (Quadro 1 e Gráfico 2).

A oferta de qualificação de pessoal de nível superior, missão da Capes e do Plano Nacional da Educação (PNE), ocorre massivamente por meio de IES públicas, assim como cerca de $90 \%$ da produção de Ciência e Tecnologia (C\&T) nacional. No Gráfico 3, é possível observar a expressividade das instituições públicas no panorama da pós-graduação brasileira, em especial as federais.

As áreas de avaliação denominadas Medicina I, Medicina II e Medicina III foram formadas por meio do agrupamento de especialidades. Complementando o Quadro 2, seguem as especificações de cada documento de área das medicinas e seus respectivos gráficos que ilustram o quantitativo de PPG por especialidade, por área.

O documento de área 2019 da Medicina I apresenta o seguinte:

A área da Medicina I possui clara característica de interdisciplinaridade. A maioria dos PPGs foram identificados como programas interdisciplinares de denominações abrangentes [...] como Cardiologia, Dermatologia, Endocrinologia, Nefrologia, Oncologia, Pneumologia, Gastronterologia/Hepatologia, entre outras (p. 5) $)^{21}$.

O Gráfico 4 - Medicina I ratifica o texto do documento de área 2019 da Medicina I, em que se observa com clareza o quantitativo de PPG com "denominações abrangentes", como "medicina". 
Gráfico 3.Distribuição dos PPG de Medicina de acordo com a natureza jurídica da IES.

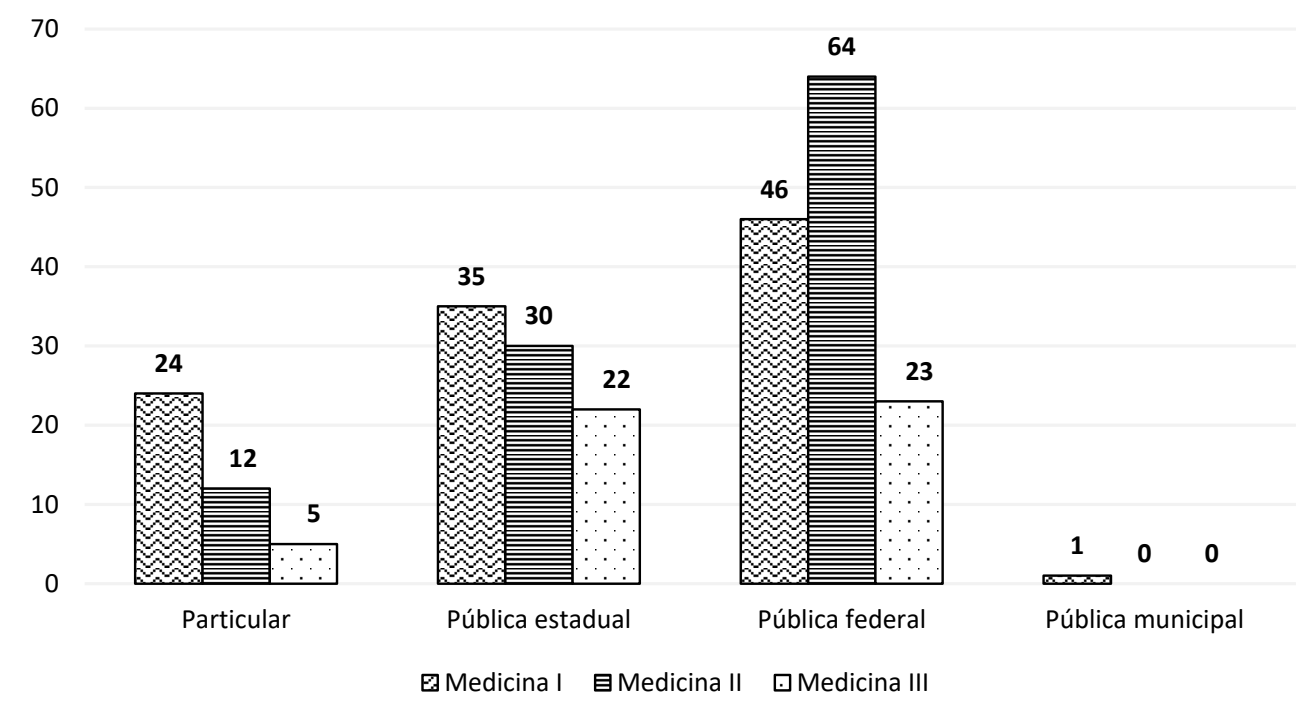

Gráfico 4.Distribuição dos PPGs, por especialidade, nas áreas avaliativas da medicina.
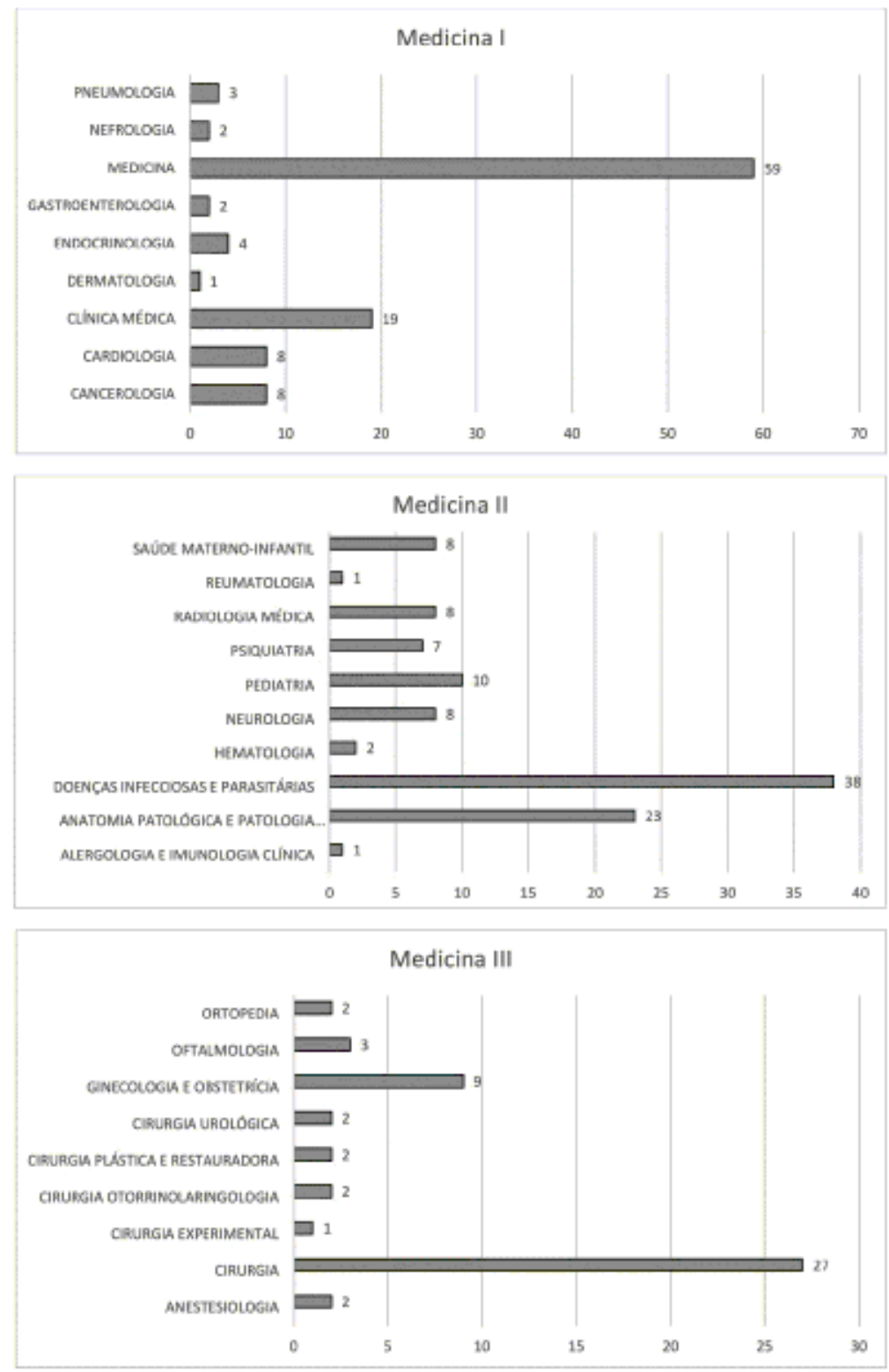
Já o documento de área de 2019 da Medicina ll apresenta o agrupamento de especialidades da seguinte forma:

A área 16 da CAPES, Medicina ll, é composta por cursos e programas voltados para a formação de pessoal qualificado em pesquisa e inovação tecnológica na área médica e da saúde ligadas a diversas especialidades médicas clínicas. [...] na área Medicina Il estão vinculados programas com atuação em algumas especialidades médicas, particularmente: 1) Doenças Infeciosas e Parasitárias/Infectologia; 2) Patologia; 3) Pediatria/Saúde Materno-Infantil; 4) Neurologia/Neurociências; 5) Psiquiatria/Saúde Mental; 6) Radiologia e Diagnóstico por Imagens; 7) Hematologia; 8) Reumatologia; 9) Alergologia. Em todos esses campos, os programas desenvolvem estudos nas áreas básicas e clínicas, muitos deles com componente experimental (p. 2) 22 .

Por fim, o documento de área 2019 da Medicina III estabelece objetivamente a seguinte delimitação:

A área Medicina III, dentro das áreas das medicinas, agrupa pesquisa com interesse na temática cirúrgica em geralenassuasespecialidades, possuindo potencial significativo para pesquisa stricto sensu, tanto nas modalidades acadêmica como na profissional (p. 1) $)^{23}$.

A partir do texto do documento de área 2019 da Medicina III, é oportuno tratar da terminologia "modalidade profissional". A modalidade profissional na pós-graduação stricto sensu brasileira foi regulamentada de "acordo com as exigências previstas na legislação - Resolução CNE/CES nº 1/2001, alterada pela Resolução CNE/CES no 24/2002"14,24,27, e as atuais legislações aplicáveis são a Portaria do Ministério da Educação (MEC) n 389/2017²5 e a Portaria Capes no 60/2019²6.

Trata-se da "capacitação de profissionais, nas diversas áreas do conhecimento, mediante o estudo de técnicas, processos ou temáticas que atendam a alguma demanda do mercado de trabalho"27, com o objetivo de "estimular atividades que respondam às necessidades técnicas/ tecnológicas do mercado de trabalho e, consequentemente, da sociedade"23. Apesar da resistência inicial das universidades e dos acadêmicos, os cursos de pós-graduação na modalidade profissional cresceram e trouxeram versatilidade para o SNPG:

A introdução do mestrado profissionalizante foi um passo que aumentou a diversidade de diplomas na pós-graduação, antes restrita aos títulos de doutorado e mestrado acadêmico. Trata-se, pois, de uma medida que aumentou a flexibilidade do sistema ${ }^{14}$.

Em junho de 2019, dos 4.590 PPGs, 18,6\% são cadastrados como profissionais, com maior incidência na área avaliativa Interdisciplinar, 94 PPGs, e na área avaliativa Administração Pública e de Empresas, Ciências Contábeis e Turismo, 80 programas.

Para as três áreas avaliativas da medicina, a incidência de cursos profissionais é bem inferior se se considerar o universo de programas: são 53 PPGs, cerca de 1,1\% do SNPG. A Medicina III realmente ganha destaque no percentil na modalidade profissional, 28\% dos seus PPGs.

Voltando para o aspecto avaliativo desse panorama, conforme consta na Portaria no 59, de 21 de março de 2017, a avaliação não é classificatória e sim comparativa, respeitando as especificidades de cada área e modalidade. As notas são conferidas a fim de estabelecer níveis de qualidade dos cursos, numa escala de 7 a 1 - muito bom, bom, regular, fraco e insuficiente -, em que "a nota 3 corresponde ao padrão mínimo de qualidade para a recomendação"19.

No caso de Programas nota 3 que possuam cursos de doutorado e de mestrado, o curso de doutorado, a critério da comissão de avaliação, poderá ser descredenciado, com a atribuição da nota 2, mantido em funcionamento o curso de mestrado, com nota $3(p .7)^{19}$.

Gráfico 5.Distribuição dos PPGs por modalidade.

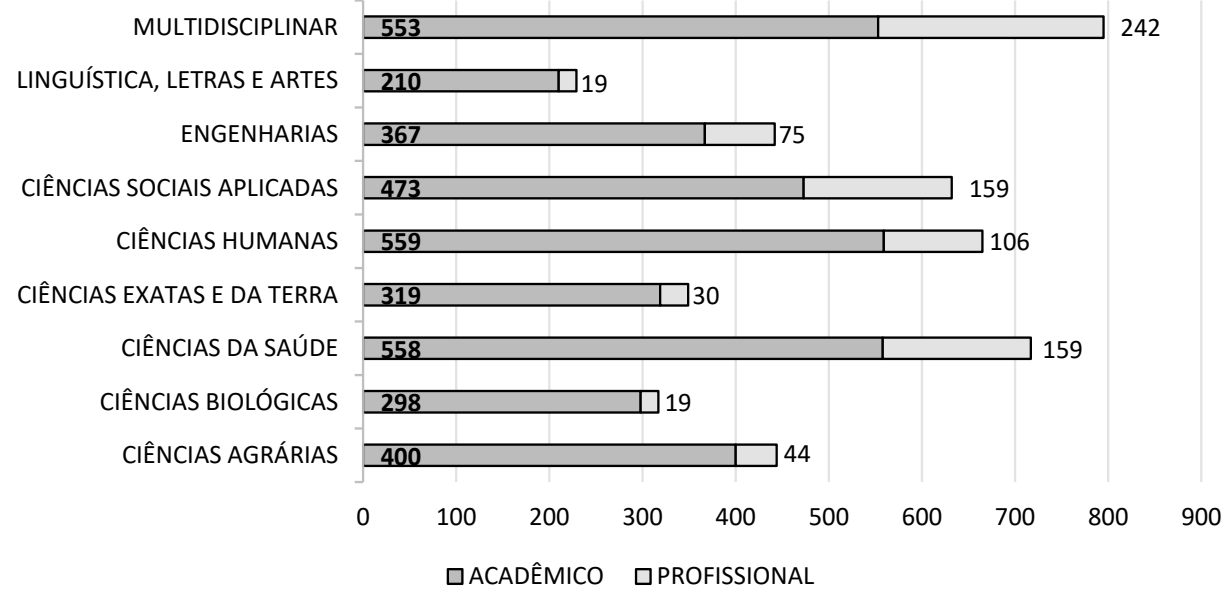


Tabela 1. PPG das três áreas avaliativas da medicina por modalidade.

\begin{tabular}{ccccc}
\hline & Medicina I & Medicina II & Medicina III & TOTAL \\
\hline Acadêmico & 82 & 91 & 36 & 209 \\
Profissional & 24 & 15 & 14 & 53 \\
TOTAL & 106 & 106 & 50 & 262 \\
\hline
\end{tabular}

O Gráfico 6 apresenta a distribuição dos PPGs das medicinas por nota e com designação " $A$ " para aqueles programas em projeto, que ainda não passaram por um período avaliativo

A transformação da educação superior a partir de 1960, em especial da graduação, causou impactos na pós-graduação em diversos pontos, em que o crescente número de IES acabou por demandar um maior contingente de pessoal de nível superior qualificado, incidindo diretamente na valoração e na expansão da pós-graduação stricto sensu.

Em 2009, o Brasil registrava 2.719 PPGs e em meados de 2019 atingiu 4.590 programas, sendo contabilizados 492 programas de excelência com padrões de qualidade internacional, notas 6 e 7. Em relação à educação médica brasileira, no recorte da pós-graduação stricto sensu, as três áreas da medicina acompanharam esse crescimento, como é demostrando nos três últimos períodos avaliativos no número de programas de pós-graduação de medicina ${ }^{28}$.

Gráfico 6. Distribuição por nota dos PPGs das medicinas.

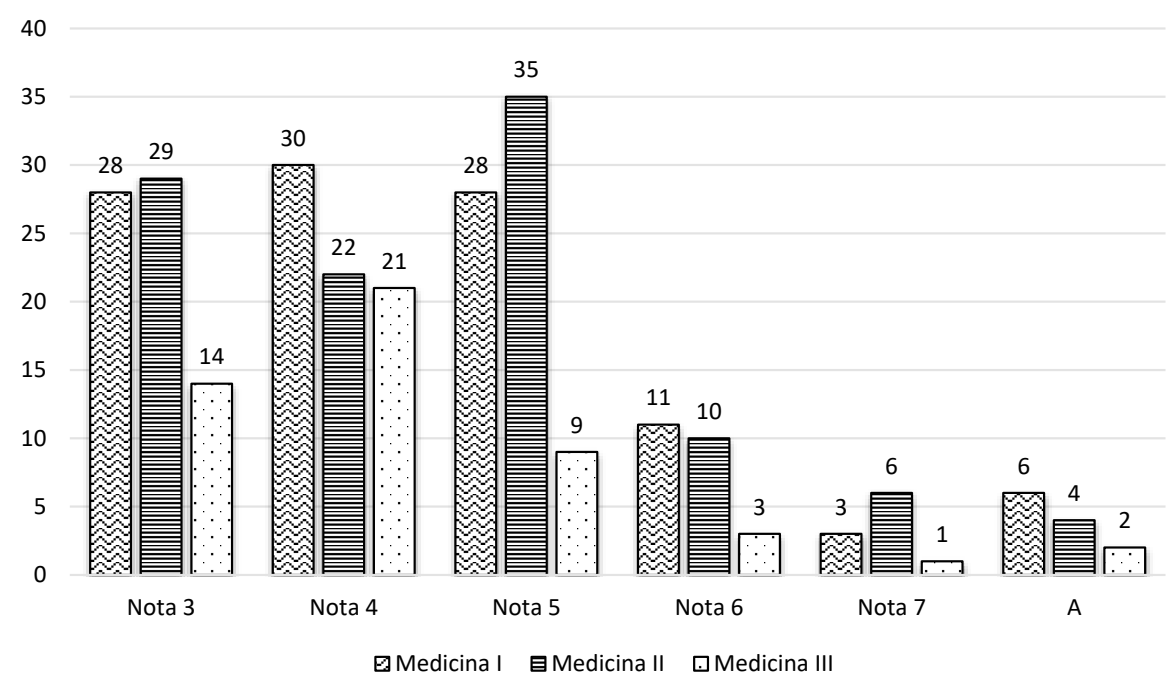

Gráfico 7. Quantitativo de PPG de cada uma das três áreas avaliativas da medicina nos últimos períodos avaliativos.

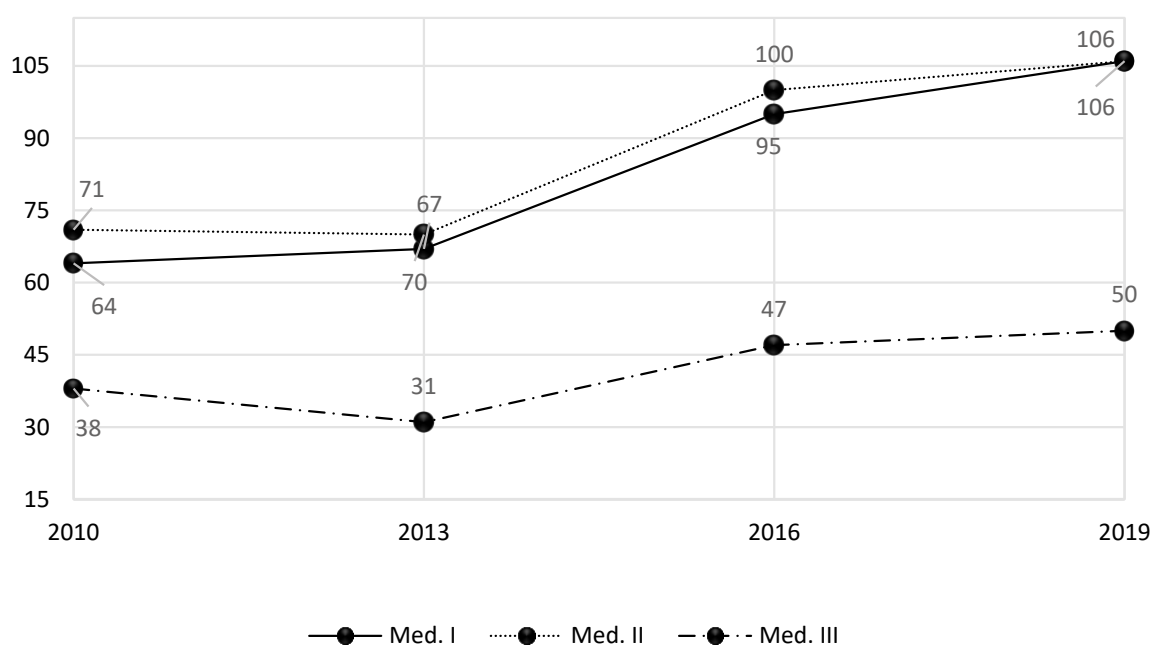


Atualmente, a pós-graduação brasileira alcança todas as UFs do país, mas ainda preserva traços de assimetria regional. São múltiplos os ângulos das assimetrias e as particularidades de um país de dimensão continental como o Brasil. No que tange à configuração geográfica, na seção "Resultados e discussão" é possível observar a formação de três polos na distribuição de PPG pelo Brasil (Figura 1).

Os estados com mais de 180 PPGs, o polo III, centralizam mais de $67 \%$ dos programas brasileiros, $62 \%$ da população brasileira e IDH médio de 0,753. Já o polo II abrange a maior porção territorial do país, $40,25 \%$, um pouco menos de um terço da população brasileira e $26 \%$ dos PPGs. O IDH dessa região é muito diversificado, indo do Pará, terceiro menor IDH do país com índice 0,646, ao maior IDH do Brasil, Distrito Federal, de 0,824. Por último, o polo I possui a menor concentração de PPG e de população do Brasil, são 285 PPGs e quase 9\% da população nacional; esse polo possui uma certa variedade de IDH, mas ainda assim apresenta o menor IDH médio dos três polos.

A comparação da distribuição geográfica dos PPGs (Figura 1) com o IDH das UFs brasileiras acaba por espelhar um país de qualidade de vida média (IDH médio de 0,710), com alta concentração da população e da pós-graduação stricto sensu, em uma faixa que corresponde a cerca de $24 \%$ da sua extensão territorial. A partir disso, destacam-se a pouca difusão e o pouco alcance das políticas públicas de desenvolvimento social. Nesse sentido, é importante identificar as fragilidades e carências de cada região a fim de trabalhar em programas de indução que venham a incrementar as faces socioeconômicas de cada UF.

Os gráficos 1 e 4 ilustram e quantificam os Quadros 1 e 2 apresentando uma ideia do todo, mas com o devido destaque para o foco deste trabalho - a medicina em suas três áreas avaliativas. Outra categorização importante abordada neste trabalho é a predominância das IES públicas na oferta de pós-graduação stricto sensu. Juntas as IES públicas, federal, estadual e municipal, são responsáveis por $88 \%$ dos PPGs brasileiros, e, no que concerne à medicina, a rede federal de ensino é predominante em todas as áreas e alcança 64 PPGs em Medicina II.

Os cursos na modalidade profissional, que outrora foram estigmatizados e até preteridos, trouxeram diversidade ao SNPG, uma vez que esses programas na "modalidade profissional podem ter essa conexão com as Instituições de Ensino Superior, mas podem, também, estar sediados em outras instituições que tenham em seu regimento objetivo de ensino e ou pesquisa" (p. 6) ${ }^{23}$. Os primeiros PPGs são datados de 1998 com apenas 27 programas, e atualmente essa modalidade vem crescendo e alcançando visibilidade, conforme mostra o Gráfico 5.

Em números absolutos, a Medicina I possui a maior oferta de PPGs profissionais: 24. Porém, a Medicina III não só apresenta o maior percentual (28\%), como também tem um claro direcionamento no documento de área para valorização dos cursos profissionais, diferentemente da Medicina I, que é composta majoritariamente de cursos acadêmicos com ênfase multidisciplinar, e da Medicina II, que abriga uma "linha de pesquisa médica mais voltada para a experimentação em laboratório ou em bancada"20.

A área Medicina III, dentro das áreas das medicinas, agrupa pesquisa com interesse na temática cirúrgica em geralenassuas especialidades, possuindo potencial significativo para pesquisa stricto sensu, tanto nas modalidades acadêmica como na profissional ( $p .1)^{23}$.

Quando se observam atentamente as notas dos programas, devem-se considerar os objetivos da avaliação, como "retratar a situação da pós-graduação brasileira no quadriênio, de forma clara e efetiva" e

[...] contribuir para o desenvolvimento de cada programa e área em particular e da pós-graduação brasileira em geral ao fornecer, a cada programa avaliado, as apreciações criteriosas sobre os pontos fortes e os pontos fracos de seu desempenho, no contexto do conjunto dos programas da área, $e$ antepor-Ihes desafios e metas para o futuro ${ }^{20}$.

Isso posto, quando se analisa o Gráfico 6 observamse números coincidentes para as áreas Medicina I e Medicina II. Ambas as áreas têm um total de 106 PPGs e $27 \%$ dos seus programas possuem nota 3 , havendo uma discreta diferenciação na contagem dos programas de nota 6 e 7. A Medicina I possui 11 programas nota 6 e três programas nota 7, juntos totalizam 13\% dos programas da área. Já a Medicina II possui dez programas nota 6 e seis programas nota 7, que juntos totalizam 15\% dos programas da área. A Medicina III é a menor área das medicinas, com apenas 50 PPGs. Desse total, 28\% possuem nota 3, 60\% estão na faixa de nota 4 e 5 , e apenas $8 \%$ receberam notas 6 e 7 na última avaliação. Observar a distribuição de notas de cada área de avaliação permite identificar não apenas os programas de pós-graduação de maior impacto no SNPG, mas também aqueles que necessitam de mais atenção para alcançar os padrões de excelência. Por meio dessa distribuição, é possível ainda embasar a formulação de políticas públicas para valorização e/ou indução de programas, conforme suas características.

Em meio ao tema da avaliação de áreas formativas, faz-se importante lembrar que a formação médica não se dá exclusivamente pelos PPGs Medicina I, II e III. No contexto do SNPG, com cursos multidisciplinares e interdisciplinares, não é incomum uma composição multiprofissional nos PPGs e nos projetos de pesquisa, no entanto essa temática é secundária ao objetivo do presente artigo.

A partir de 2021, está prevista uma reestruturação da 
avaliação da pós-graduação promovida pela Capes. Asmudanças serão implementadas a partir do próximo período avaliativo, com resultados previstos para 2025. A nova estrutura avaliativa deve considerar "cinco dimensões: Ensino e Aprendizagem, Produção de Conhecimento, Inserção Internacional e Regional, Inovação e Transferência do Conhecimento e Impacto na Sociedade" (p. 29) ${ }^{29}$, a fim de construir um cenário avaliativo mais consistente.

Por fim, fica a perspectiva de que, para além do número de PPG e de suas respectivas notas, um panorama qualitativo deve considerar indicadores como produção científica e impacto social, e mais tantos outros aspectos da pós-graduação stricto sensu brasileira, mas esses indicadores são temas a serem abordados em estudos futuros, utilizando-se dados e ferramentas metodológicas condizentes.

\section{CONTRIBUIÇÃO DAS AUTORAS}

Hayslla Boaventura Piotto participou da investigação, da escrita do rascunho e da edição da versão original. Luciana Calabró participou da supervisão.

\section{CONFLITO DE INTERESSES}

Declaram os não haver conflito de interesses neste estudo.

\section{FINANCIAMENTO}

Declaramos que não houve financiamento nesta pesquisa.

\section{REFERÊNCIAS}

1. Machado $C D B$, Wuo A, Heinzle M. Educação médica no Brasil: uma análise histórica sobre a formação acadêmica e pedagógica. Rev Bras Educ Med. 2018;42:66-73 [acesso em 23 jun 2019]. Disponível em: http://www.scielo. br/pdf/rbem/v42n4/1981-5271-rbem-42-4-0066.pdf.

2. Neves NMBC, Neves FBCS, Bitencourt AGV. O ensino médico no Brasil: origens e transformações. Gaz Med Bahia. 2005;75:162-8 [acesso em 29 jun 2019]. Disponível em: http://www.gmbahia.ufba.br/index.php/ gmbahia/article/viewFile/362/351.

3. Brasil. Plano Nacional de Pós-Graduação 2011-2020. Brasília: Capes; 2010. $262 \mathrm{p}$.

4. Brasil. Decreto no 19.851, de 11 abril de 1931. Dispõe que o ensino superior no Brasil obedecerá, de preferência, ao sistema universitário, podendo ainda ser ministrado em institutos isolados. Diário Oficial da União; 15 abr 1931.

5. Brasil. Decreto no 19.852, de 11 de abril de 1931. Dispõe sobre a organização da Universidade do Rio de Janeiro. Diário Oficial da União; 11 abr 1931.

6. Sucupira N. Antecedentes e primórdios da pós-graduação. 1980 [acesso em $1^{\circ}$ jul 2019]. Disponível em: http://bibliotecadigital.fgv.br/ojs/index. php/fe/article/viewFile/60545/58792.

7. Brasil. Decreto $n^{\circ} 21.321$, de 18 de junho de 1946. Aprova o Estatuto da Universidade do Brasil. Diário Oficial da União; 20 jun 1946.

8. Gouvêa F, Mendonça AWPC. A contribuição de Anísio Teixeira para a institucionalização da pós-graduação no Brasil: um percurso com os boletins da CAPES. Perspectiva. 2006;24:111-32. [acesso em 10 jul 2019]. Disponível em: https://periodicos.ufsc.br/index.php/perspectiva/article/ view/10314/9577.

9. Brasil. Lei no 4.024, de 20 de dezembro de 1961. Fixa as Diretrizes e Bases da Educação Nacional. Diário Oficial da União; 27 dez 1961.
10. Almeida Junior A. Parecer CFE no 977/65, aprovado em 3 dez. 1965. Rev Bras Educ. 2005;(30):162-73 [acesso em 11 fev 2020]. Disponível em: http://www.scielo.br/pdf/rbedu/n30/a14n30.pdf.

11. Silveira MMRV, Rocha-Neto I. O processo de avaliação do Sistema Nacional de Pós-Graduação: identifica a CAPES e a diferença das outras Agências. Poiésis: Revista do Programa de Pós-Graduação em Educação. 2016;10:292-310 [acesso em 11 fev 2020]. Disponível em: http://www. portaldeperiodicos.unisul.br/index.php/Poiesis/article/view/1961.

12. Brasil. Lei $n^{\circ} 8.405$, de 9 de janeiro de 1992. Autoriza o Poder Executivo a instituir como fundação pública a Coordenação de Aperfeiçoamento de Pessoal de Nível Superior (Capes) e dá outras providências. Diário Oficial da União; 10 jan 1992.

13. Instituto Brasileiro de Geografia e Estatística. População [acesso em 16 ago 2020]. Disponível em: https://cidades.ibge.gov.br/brasil/panorama.

14. Steiner JE. Qualidade e diversidade institucional na pós-graduação brasileira. Estud Av. 2005; 19(54):341-65 [acesso em 20 ago 2020]. Disponível em: https:// www.scielo.br/scielo.php?script=sci_arttext\&pid=S0103-40142005000200019.

15. Brasil. Lei no 11.502, de 11 de julho de 2007. Modifica as competências e a estrutura organizacional da fundação Coordenação de Aperfeiçoamento de Pessoal de Nível Superior - CAPES, de que trata a Lei $n^{\circ} 8.405$, de 9 de janeiro de 1992; e altera as Leis nos. 8.405, de 9 de janeiro de 1992, e 11.273, de 6 de fevereiro de 2006. Diário Oficial da União; 12 jul 2007.

16. Brasil. Resolução CNS/CES n 1, de 3 de abril de 2001. Estabelece normas para o funcionamento de cursos de pós-graduação. Diário Oficial da União; 9 abr 2001

17. Coordenação de Aperfeiçoamento de Pessoal de Nível Superior. Portaria no 13, de 01 de abril de 2002. Dispõe sobre as notas atribuídas aos programas de pós-graduação nos procedimentos do sistema de avaliação e no funcionamento de curso de mestrado e doutorado. Diário Oficial da União; $1^{\circ}$ abr 2019.

18. Coordenação de Aperfeiçoamento de Pessoal de Nível Superior. Portaria no 99, de 21 de dezembro de 2005. Institui a coleta de dados e elaboração de estatísticas sobre o reconhecimento no Brasil de títulos de mestres e doutores outorgados por instituições estrangeiras. Diário Oficial da União; 21 dez 2005.

19. Coordenação de Aperfeiçoamento de Pessoal de Nível Superior. Portaria $n^{\circ}$ 59, de 21 de março de 2017. Dispõe sobre o regulamento da Avaliação Quadrienal. Diário Oficial da União; 27 mar 2017.

20. Coordenação de Aperfeiçoamento de Pessoal de Nível Superior. Sobre a avaliação [acesso em 17 fev 2020]. Disponível em: https://www.CAPES. gov.br/avaliacao/sobre-a-avaliacao.

21. Coordenação de Aperfeiçoamento de Pessoal de Nível Superior. Documento de área. Área 15: Medicina I [acesso em 17 fev 2020]. Disponível em https://www.CAPES.gov.br/images/Documento_de_\%C3\%A1rea_2019/ Medicina_I.pdf.

22. Coordenação de Aperfeiçoamento de Pessoal de Nível Superior. Documento de área. Área 16: Medicina II [acesso em 17 fev 2020]. Disponível em https://www.CAPES.gov.br/images/Documento_de_\%C3\%A1rea_2019/ MEDICINA_II.pdf.

23. Coordenação de Aperfeiçoamento de Pessoal de Nível Superior. Documento de área. Área 17: Medicina III [acesso em 17 fev 2020]. Disponível em: https:// www.CAPES.gov.br/images/Documento_de_\%C3\%A1rea_2019/MED3.pdf.

24. Brasil. Resolução $n^{\circ} 24$, de 18 de dezembro de 2002. Altera a redação do parágrafo $4^{\circ}$ do artigo $1^{\circ} \mathrm{e} o$ artigo $2^{\circ}$, da Resolução CNE/CES 1/2001, que estabelece normas para o funcionamento de cursos de pós-graduação. Diário Oficial da União; 20 dez 2002.

25. Coordenação de Aperfeiçoamento de Pessoal de Nível Superior. Portaria no 389, de 23 de março de 2017. Dispõe sobre o mestrado e doutorado profissional no âmbito da pós-graduação stricto sensu. Diário Oficial da União; 24 mar 2017.

26. Coordenação de Aperfeiçoamento de Pessoal de Nível Superior. Portaria $\mathrm{n}^{\circ}$ 60, de 20 de março de 2019. Dispõe sobre o mestrado e doutorado profissionais, no âmbito da Coordenação de Aperfeiçoamento de Pessoal de Nível Superior - CAPES. Diário Oficial da União; 22 mar 2019. 
27. Coordenação de Aperfeiçoamento de Pessoal de Nível Superior. Mestrado profissional: o que é? [acesso em 17 fev 2020]. Disponível em: https:// www.CAPES.gov.br/avaliacao/sobre-a-avaliacao/mestrado-profissionalo-que-e.

28. Coordenação de Aperfeiçoamento de Pessoal de Nível Superior. GEOCAPES [acesso em $17 \mathrm{fev}$ 2020]. Disponível em: https://geoCAPES. CAPES.gov.br/geoCAPES/.
29. Marques F. Avaliação em 5 dimensões. Rev Pesq FAPESP. 2019;286:28-31 [acesso em 12 fev 2020]. Disponível em: https://revistapesquisa.fapesp.br/ avaliacao-em-5-dimensoes/. 